International Journal of Pure and Applied Mathematics

Volume 93 No. 4 2014, 567-580

ISSN: 1311-8080 (printed version); ISSN: 1314-3395 (on-line version)

url: http://www.ijpam.eu

doi: http://dx.doi.org/10.12732/ijpam.v93i4.7

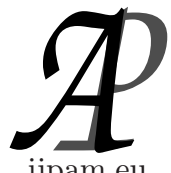

ijpam.eu

\title{
ON SUBMANIFOLDS OF A RIEMANNIAN MANIFOLD WITH A SEMI-SYMMETRIC RECURRENT-METRIC CONNECTION
}

\author{
Jaeman Kim \\ Department of Mathematics Education \\ Kangwon National University \\ Chunchon 200-701, Kangwon Do, KOREA
}

\begin{abstract}
We study some properties of submanifolds of a Riemannian manifold with a semi-symmetric recurrent-metric connection. Among others, the Gauss equation, the Codazzi-Mainardi equation and the Ricci equation for such a connection have been derived.
\end{abstract}

AMS Subject Classification: 53A30, 53B35, 53C25, 53C55, 53C56 Key Words: submanifolds, semi-symmetric recurrent-metric connection, curvature with respect to semi-symmetric recurrent-metric connection, Gauss equation, Codazzi-Mainardi equation, Ricci equation, totally geodesic, totally umbilical, minimal, sectional curvature with respect to semi-symmetric recurrentmetric connection

\section{Introduction}

Let $M=(M, g)$ be a Riemannian manifold of dimension $n$ with a metric tensor g. A linear connection $\nabla$ on $M$ satisfies

$$
(i) \nabla_{f X+g Y} Z=f \nabla_{X} Z+g \nabla_{Y} Z,(i i) \nabla_{X}(f Y)=(X f) Y+f \nabla_{X} Y,
$$

where $f, g$ are smooth functions on $M$ and $X, Y, Z$ are smooth vector fields on 
$M$. The torsion tensor $T$ of $\nabla$ is given by

$$
T(X, Y)=\nabla_{X} Y-\nabla_{Y} X-[X, Y] .
$$

If the torsion tensor $T$ vanishes, then $\nabla$ is called symmetric, otherwise it is nonsymmetric. If the metric tensor $g$ of $M$ satisfies $\nabla g=0$, then $\nabla$ is called metric, otherwise it is nonmetric. It is well known that the Levi-Civita connection is the only linear connection which is both symmetric and metric. In particular, a nonsymmetric connection is said to be semi-symmetric if its torsion tensor $T$ is of the form

$$
T(X, Y)=u(Y) X-u(X) Y,
$$

where $u$ is a 1-form on $M$.

The idea of semi-symmetric metric connection on a Riemannian manifold was introduced by Yano and some of its properties were studied in [14]. A hypersurface of a Riemannian manifold with a semi-symmetric metric connection was studied by Imai [6]. Later in [9] Nakao investigated submanifolds of a Riemannian manifold with the semi-symmetric metric connection. On the other hand, Agashe and Chafle [1] introduced the idea of a semi-symmetric nonmetric connection on a Riemannian manifold and they [2] sequently studied submanifolds of a Riemannian manifold with the semi-symmetric nonmetric connection mentioned in [1]. Other types of semi-symmetric nonmetric connections were introduced by Sengupta, De and Binh [12]; Sengupta and De [11]. Later submanifolds of Riemannian manifolds with the semi-symmetric nonmetric connections defined in [11,12] were studied by Ozgur [10] and Dogru [5], respectively. On the other hand, a different kind of semi-symmetric nonmetric connection (namely, semi-symmetric recurrent-metric connection) was defined and extensively studied by Andonie and Smaranda [3]; Liang [8]. Considering these aspects, we are motivated to study submanifolds of a Riemannian manifold with the semi-symmetric recurrent-metric connection mentioned in $[3,8]$. More precisely, in Section 2, a general description of Riemannian manifold and its submanifold is given and then a semi-symmetric recurrent-metric connection is defined. In Section 3, we show that the induced connection on a submanifold of a Riemannian manifold with the semi-symmetric recurrent-metric connection is also a semi-symmetric recurrent-metric connection. And then the Gauss, the Codazzi-Mainardi and the Ricci equations for such a connection have been derived. We also consider the totally geodesic, totally umbilical and minimal submanifolds of a Riemannian manifold with the semi-symmetric recurrentmetric connection. Finally, a concrete example of submanifold of a Riemannian manifold with the semi-symmetric recurrent-metric connection is given. 


\section{Preliminaries}

Let $M$ be an $n$-dimensional submanifold of an $(n+l)$-dimensional Riemannian manifold $\widetilde{M}$. From now on, $g$ refers to the Riemannian metric tensor on $\widetilde{M}$ as well as the induced one on $M$. Also, in the sequel, $\widetilde{X}, \widetilde{Y}, \widetilde{Z}, \widetilde{W}$ denote the vector fields on $\widetilde{M} ; X, Y, Z, W$ denote the vector fields tangent to $M$. The formulas of Gauss and Weingarten are given by

$$
\widetilde{\nabla}_{X} Y=\nabla_{X} Y+h(X, Y)
$$

and

$$
\widetilde{\nabla}_{X} \eta=-A_{\eta} X+\nabla_{X}{ }^{\perp} \eta
$$

respectively, where $\eta$ is a normal vector field of $M$ in $\widetilde{M}$ and $\nabla$ is the induced Riemannian connection on $M$ from the Riemannian connection $\widetilde{\nabla}$ on $\widetilde{M}$ and $\nabla^{\perp}$ is a (metric) connection in the normal bundle $T(M)^{\perp}$ with respect to the fibre metric induced from $g$ [7]. Note that the second fundamental form $h$ is related to the shape operator $A_{\eta}$ by

$$
g(h(X, Y), \eta)=g\left(A_{\eta} X, Y\right)=g\left(X, A_{\eta} Y\right) .
$$

If $h=0$, then $M$ is said to be totally geodesic. The mean curvature vector $H$ of $M$ is given by

$$
H=\frac{1}{n} \text { traceh }
$$

$M$ is said to be minimal if $H=0 ; M$ is said to be totally umbilical if $h(X, Y)=$ $g(X, Y) H$. The covariant derivative of $h$ is defined by

$$
\left(\widehat{\nabla}_{X} h\right)(Y, Z)=\nabla_{X}^{\perp}(h(Y, Z))-h\left(\nabla_{X} Y, Z\right)-h\left(Y, \nabla_{X} Z\right) .
$$

The connection $\widehat{\nabla}$ is called the van der Waerden-Bortolotti connection of $M$ [4]. In [13], a linear connection $\tilde{\nabla}$ on a Riemannian manifold $\widetilde{M}$ is given by

$$
\tilde{\nabla}_{\widetilde{X}} \widetilde{Y}=\widetilde{\nabla}_{\widetilde{X}} \widetilde{Y}+u_{1}(\widetilde{Y}) \widetilde{X}-g(\widetilde{X}, \widetilde{Y}) U_{1}-u_{2}(\widetilde{X}) \widetilde{Y}-u_{2}(\widetilde{Y}) \widetilde{X}+g(\widetilde{X}, \widetilde{Y}) U_{2},
$$

where $u_{1}$ and $u_{2}$ are 1 -forms associated with the vector fields $U_{1}$ and $U_{2}$ on $\widetilde{M}$ by $u_{1}(\widetilde{X})=g\left(U_{1}, \widetilde{X}\right)$ and $u_{2}(\widetilde{X})=g\left(U_{2}, \widetilde{X}\right)$, respectively. Using (2.5), the torsion tensor $\tilde{T}$ of $\tilde{\nabla}$ is given by

$$
\dot{\widetilde{T}}(\widetilde{X}, \widetilde{Y})=u_{1}(\widetilde{Y}) \widetilde{X}-u_{1}(\widetilde{X}) \widetilde{Y} .
$$


Furthermore, using (2.5), we get

$$
\left(\dot{\widetilde{\nabla}}_{\widetilde{X}} g\right)(\widetilde{Y}, \widetilde{Z})=2 u_{2}(\widetilde{X}) g(\widetilde{Y}, \widetilde{Z}) .
$$

Therefore, the linear connection $\tilde{\nabla}$ defined by $(2.5)$ is adequate to be called a semi-symmetric recurrent-metric connection [13]. From now on, for the sake of simplicity, a semi-symmetric recurrent-metric connection is briefly denoted by a SSRM connection. We define the curvature tensor of type $(1,3)$ of a SSRM connection $\tilde{\nabla}$ by

$$
\dot{\widetilde{R}}(\widetilde{X}, \widetilde{Y}) \widetilde{Z}=\dot{\widetilde{\nabla}}_{\widetilde{X}} \tilde{\nabla}_{\widetilde{Y}} \widetilde{Z}-\dot{\widetilde{\nabla}}_{\widetilde{Y}} \tilde{\nabla}_{\widetilde{X}} \widetilde{Z}-\dot{\nabla}_{[\widetilde{X}, \widetilde{Y}]} \widetilde{Z}
$$

Also, the curvature tensor of type $(0,4)$ of the SSRM connection $\tilde{\nabla}$ is defined by

$$
\tilde{R}(\widetilde{X}, \widetilde{Y}, \widetilde{Z}, \widetilde{W})=g(\tilde{R}(\widetilde{X}, \widetilde{Y}) \widetilde{Z}, \widetilde{W}) .
$$

\section{Submanifolds of a Riemannian Manifold with a Semi-Symmetric Recurrent-Metric Connection}

Let $\dot{\nabla}$ be the induced connection on $M$ from the SSRM connection $\tilde{\nabla}$ on $\widetilde{M}$ by the equation which may be called the formula of Gauss with respect to the SSRM connection

$$
\dot{\widetilde{\nabla}}_{X} Y=\dot{\nabla}_{X} Y+\dot{h}(X, Y)
$$

where $\hat{h}$ is a normal bundle valued tensor of type $(0,2)$. Taking account of $(2.1)$, (2.5) and (3.10), we have

$$
\begin{gathered}
\dot{\nabla}_{X} Y+\dot{h}(X, Y)=\nabla_{X} Y+h(X, Y)+u_{1}(Y) X-g(X, Y) U_{1}^{\top}-g(X, Y) U_{1}^{\perp} \\
-u_{2}(X) Y-u_{2}(Y) X+g(X, Y) U_{2}^{\top}+g(X, Y) U_{2}^{\perp},
\end{gathered}
$$

which yields

$$
\dot{\nabla}_{X} Y=\nabla_{X} Y+u_{1}(Y) X-g(X, Y) U_{1}^{\top}-u_{2}(X) Y-u_{2}(Y) X+g(X, Y) U_{2}^{\top}
$$

and

$$
\hat{h}(X, Y)=h(X, Y)-g(X, Y) U_{1}^{\perp}+g(X, Y) U_{2}^{\perp} .
$$

Here we denote by $U_{i}^{\top}$ and $U_{i}^{\perp}$ the tangential and normal components of $U_{i}(i=$ $1,2)$, respectively. It follows from (3.11) and the properties of a Riemannian connection $\nabla$ that

$$
\dot{T}(X, Y)=\dot{\nabla}_{X} Y-\dot{\nabla}_{Y} X-[X, Y]=u_{1}(Y) X-u_{1}(X) Y
$$


and

$$
\left(\dot{\nabla}_{X} g\right)(Y, Z)=X(g(Y, Z))-g\left(\dot{\nabla}_{X} Y, Z\right)-g\left(Y, \dot{\nabla}_{X} Z\right)=2 u_{2}(X) g(Y, Z)
$$

Therefore, we obtain the following:

Theorem 3.1. The induced connection $\dot{\nabla}$ on a submanifold $M$ of a Riemannian manifold $\widetilde{M}$ with the SSRM connection $\widetilde{\nabla}$ is also a SSRM connection.

Analogous to the definition of the mean curvature vector $H$ of $M$, we define the mean curvature vector $\dot{H}$ of $M$ with respect to the SSRM connection $\tilde{\widetilde{\nabla}}$ by

$$
\dot{H}=\frac{1}{n} \text { tracé́h. }
$$

$M$ is said to be minimal with respect to the SSRM connection $\dot{\widetilde{\nabla}}$ if $\dot{H}=0 ; M$ is said to be totally geodesic with respect to the SSRM connection $\tilde{\nabla}$ if $\dot{h}=0$ ; $M$ is said to be totally umbilical with respect to the SSRM connection $\dot{\nabla}$ if $\hat{h}(X, Y)=g(X, Y) \dot{H}$. Now we can state the following:

Theorem 3.2. Let $M$, be a submanifold of a Riemannian manifold $\widetilde{M}$ with the SSRM connection $\widetilde{\nabla}$. Then $M$ is totally umbilical if and only if $M$ is

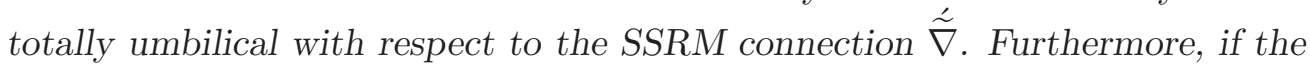
associated vector fields $U_{1}$ and $U_{2}$ are tangent to $M$, then:

(i) the second fundamental form $h$ of $M$ and the second fundamental form $\dot{h}$ of $M$ with respect to the SSRM connection $\dot{\nabla}$ coincide;

(ii) the mean curvature vector $H$ of $M$ and the mean curvature vector $H$ of $M$ with respect to the SSRM connection $\stackrel{\tilde{\nabla}}{\text { coincide; }}$

(iii) $M$ is totally geodesic if and only if $M$ is totally geodesic with respect to the SSRM connection $\dot{\nabla}$;

(iv) $M$ is minimal if and only if $M$ is minimal with respect to the SSRM connection $\stackrel{\dot{\nabla}}{\nabla}$.

Proof. In view of (3.12) and (3.15), we have

$$
\dot{H}=H-U_{1}^{\perp}+U_{2}^{\perp} \text {. }
$$

Comparing the above relation with (3.12), we conclude that a totally umbilical $M$ is also totally umbilical with respect to $\tilde{\nabla}$, and vice versa. On the other hand, let us assume that $U_{1}$ and $U_{2}$ are tangent to $M$. Then we have from (3.12)

$$
\hat{h}(X, Y)=h(X, Y) .
$$


Taking account of the above identity and (3.15), we obtain that (i), (ii), (iii) and (iv) hold true. This completes the proof of theorem 3.2.

Let $\eta$ be a normal vector field of $M$ in $\widetilde{M}$. Taking account of (2.5), we get

$$
\tilde{\widetilde{\nabla}}_{X} \eta=\widetilde{\nabla}_{X} \eta+u_{1}(\eta) X-u_{2}(X) \eta-u_{2}(\eta) X,
$$

which yields from $(2.2)$

$$
\tilde{\widetilde{\nabla}}_{X} \eta=-A_{\eta} X+\nabla_{X}^{\perp} \eta+u_{1}(\eta) X-u_{2}(X) \eta-u_{2}(\eta) X .
$$

From (3.17), we can define a tensor of type $(1,1)$ on $M$ as follows:

$$
\hat{A}_{\eta}=A_{\eta}-u_{1}(\eta) I d+u_{2}(\eta) I d .
$$

Taking (3.17) and (3.18) into account, we have

$$
\tilde{\nabla}_{X} \eta=-\dot{A}_{\eta} X+\nabla \frac{\perp}{X} \eta-u_{2}(X) \eta,
$$

which may be called the formula of Weingarten with respect to the SSRM connection $\tilde{\nabla}$. Now we obtain the following:

Theorem 3.3. If $M$ is a submanifold of a Riemannian manifold $\widetilde{M}$ with the SSRM connection $\stackrel{\nabla}{\nabla}$, then for the unit normal vector field $\eta$ of $M$ in $\widetilde{M}$, the principal directions and the principal directions with respect to the SSRM connection $\dot{\nabla}$ coincide. Moreover, if the associated vector field $U_{1}$ and $U_{2}$ are tangent to $M$, then the principal curvatures are equal to the principal curvatures with respect to the SSRM connection $\tilde{\nabla}$.

Proof. Taking account of (3.18), we conclude that the principal directions and the principal directions with respect to the SSRM connection $\tilde{\nabla}$ coincide. On the other hand, let us assume that $U_{1}$ and $U_{2}$ are tangent to $M$. Then we have from (3.18)

$$
\hat{A}_{\eta}=A_{\eta}
$$

which implies that the principal curvatures are equal to the principal curvatures with respect to $\tilde{\nabla}$. This completes the proof of theorem 3.3 .

Theorem 3.4. Let $M$, be a submanifold of a Riemannian manifold $\widetilde{M}$ with the SSRM connection $\widetilde{\nabla}$. Then the shape operators are simultaneously diagonalizable if and only if the shape operators with respect to the SSRM connection $\dot{\nabla}$ are simultaneously diagonalizable. 
Proof. For the unit normal vector fields $\eta, \mu$ of $M$ in $\widetilde{M}$, we have from $(3.18)$

$$
g\left(\hat{A}_{\eta} X, Y\right)=g\left(X, \dot{A}_{\eta} Y\right)
$$

and

$$
\begin{aligned}
& g\left(\left[\dot{A}_{\eta}, \dot{A}_{\mu}\right] X, Y\right)=g\left(\left(\dot{A}_{\eta} \dot{A}_{\mu}-\dot{A}_{\mu} \dot{A}_{\eta}\right) X, Y\right) \\
= & g\left(\left(A_{\eta} A_{\mu}-A_{\mu} A_{\eta}\right) X, Y\right)=g\left(\left[A_{\eta}, A_{\mu}\right] X, Y\right),
\end{aligned}
$$

which gives the required result.

Let $R$ be the curvature tensor with respect to the induced SSRM connection $\dot{\nabla}$ on a submanifold $M$. More precisely,

$$
\dot{R}(X, Y) Z=\dot{\nabla}_{X} \dot{\nabla}_{Y} Z-\dot{\nabla}_{Y} \dot{\nabla}_{X} Z-\dot{\nabla}_{[X, Y]} Z
$$

Taking (3.10) and (3.19) into account, we have

$$
\begin{gathered}
\dot{\widetilde{R}}(X, Y) Z=\dot{R}(X, Y) Z+\dot{h}\left(X, \dot{\nabla}_{Y} Z\right)-\dot{h}\left(Y, \dot{\nabla}_{X} Z\right) \\
-\dot{h}([X, Y], Z)-\dot{A}_{\dot{h}(Y, Z)}(X)+\dot{A}_{\dot{h}(X, Z)}(Y)+\nabla_{X}^{\perp} \dot{h}(Y, Z) \\
-\nabla_{Y}^{\perp} \dot{h}(X, Z)-u_{2}(X) \hat{h}(Y, Z)+u_{2}(Y) \dot{h}(X, Z) .
\end{gathered}
$$

From (2.3), (3.12) and (3.18), it follows that

$$
\begin{gathered}
\tilde{R}(X, Y, Z, W)=\dot{R}(X, Y, Z, W)-g(h(X, W), h(Y, Z)) \\
+u_{1}(h(X, W)) g(Y, Z)-u_{2}(h(X, W)) g(Y, Z)+g(h(X, Z), h(Y, W)) \\
-u_{1}(h(Y, W)) g(X, Z)+u_{2}(h(Y, W)) g(X, Z)+u_{1}(h(Y, Z)) g(X, W) \\
-u_{1}\left(U_{1}^{\perp}\right) g(X, W) g(Y, Z)+u_{1}\left(U_{2}^{\perp}\right) g(X, W) g(Y, Z)-u_{1}(h(X, Z)) g(Y, W) \\
+u_{1}\left(U_{1}^{\perp}\right) g(X, Z) g(Y, W)-u_{1}\left(U_{2}^{\perp}\right) g(X, Z) g(Y, W)-u_{2}(h(Y, Z)) g(X, W) \\
+u_{2}\left(U_{1}^{\perp}\right) g(X, W) g(Y, Z)-u_{2}\left(U_{2}^{\perp}\right) g(X, W) g(Y, Z)+u_{2}(h(X, Z)) g(Y, W) \\
-u_{2}\left(U_{1}^{\perp}\right) g(X, Z) g(Y, W)+u_{2}\left(U_{2}^{\perp}\right) g(X, Z) g(Y, W),
\end{gathered}
$$

which may be called the Gauss equation with respect to the SSRM connection. The manifold $\widetilde{M}$ (resp. $M$ ) with a SSRM connection $\tilde{\nabla}$ (resp. $\dot{\nabla}$ ) is said to be $\dot{\widetilde{\nabla}}$-flat (resp. $\dot{\nabla}$-flat) if the curvature tensor $\dot{\widetilde{R}}$ (resp. $\dot{R}$ ) of $\dot{\widetilde{\nabla}}$ (resp. $\dot{\nabla}$ ) vanishes. Now we can state the following: 
Theorem 3.5. Let $\widetilde{M}$ be a $\tilde{\nabla}$-flat manifold. If $M$ is totally geodesic and the associated vector fields $U_{1}, U_{2}$ are tangent to $M$, then $M$ is a $\dot{\nabla}$-flat manifold.

Proof. From $h=0, U_{i}^{\perp}=0(i=1,2)$ and (3.21), it follows that the theorem holds.

Concerning the sectional curvature with respect to the SSRM connection, we have the following:

Theorem 3.6., Let $M$ be a submanifold of a Riemannian $\widetilde{M}$ with the SSRM connection $\stackrel{\sim}{\nabla}$.

(i) If the associated vector fields $U_{1}, U_{2}$ are tangent to $M$, then for orthonormal tangent vector fields $X, Y$ on $M$, we have

$$
\stackrel{\tilde{K}}{(X, Y)}=\dot{K}(X, Y)+g(h(X, Y), h(X, Y))-g(h(X, X), h(Y, Y)) .
$$

(ii) Moreover, if $\alpha$ is a geodesic curve of $\widetilde{M}$ which lies in $M$ and $X$ is the unit tangent vector field of $\alpha$ in $M$, then we have

$$
\dot{\widetilde{K}}(X, Y) \geq \dot{K}(X, Y) .
$$

(iii) Furthermore, if $Y$ is parallel along $\alpha$ in $\widetilde{M}$, then we have

$$
\stackrel{\tilde{K}}{(X, Y)}=\dot{K}(X, Y)
$$

Proof. Suppose that $X=W, Y=Z$ are orthonormal tangent vector fields on $M$. Then we have from (3.21)

$$
\begin{gathered}
\dot{\widetilde{K}}(X, Y)=\dot{K}(X, Y)-g(h(X, X), h(Y, Y))+u_{1}(h(X, X)) \\
-u_{2}(h(X, X))+g(h(X, Y), h(X, Y))+u_{1}(h(Y, Y))-u_{1}\left(U_{1}^{\perp}\right) \\
+u_{1}\left(U_{2}^{\perp}\right)-u_{2}(h(Y, Y))+u_{2}\left(U_{1}^{\perp}\right)-u_{2}\left(U_{2}^{\perp}\right) .
\end{gathered}
$$

Since $U_{1}, U_{2}$ are tangent to $M$, the last relation reduces to

$$
\dot{\widetilde{K}}(X, Y)=\dot{K}(X, Y)+g(h(X, Y), h(X, Y))-g(h(X, X), h(Y, Y))
$$

Let $\alpha$ be a geodesic in $\widetilde{M}$ which lies in $M$ and $X$ be a unit tangent vector field of $\alpha$ in $M$. Then we have from (2.1)

$$
h(X, X)=0 .
$$


Therefore, from (3.22) it follows that

$$
\stackrel{\tilde{K}}{(X, Y)}=\dot{K}(X, Y)+g(h(X, Y), h(X, Y))
$$

If we assume $\widetilde{\nabla}_{X} Y=0$, then we have from (2.1)

$$
\nabla_{X} Y=h(X, Y)=0
$$

which yields from (3.23)

$$
\stackrel{\tilde{K}}{(X, Y)}=\dot{K}(X, Y)
$$

This completes the proof of theorem 3.6.

In view of $(3.13),(3.18)$ and $(3.20)$, the normal component of $\widetilde{R}(X, Y) Z$ is obtained as follows:

$$
\begin{gathered}
(\dot{\widetilde{R}}(X, Y) Z)^{\perp}=\dot{h}\left(X, \dot{\nabla}_{Y} Z\right)-\dot{h}\left(Y, \dot{\nabla}_{X} Z\right) \\
-\dot{h}([X, Y], Z)+\nabla_{X}^{\perp} \dot{h}(Y, Z)-\nabla_{Y}^{\perp} \dot{h}(X, Z) \\
-u_{2}(X) \dot{h}(Y, Z)+u_{2}(Y) \dot{h}(X, Z) \\
=\left(\hat{\nabla}_{X} \hat{h}\right)(Y, Z)-\left(\hat{\nabla}_{Y} \dot{h}\right)(X, Z)-u_{1}(X) \hat{h}(Y, Z) \\
+u_{1}(Y) \hat{h}(X, Z)-u_{2}(X) \hat{h}(Y, Z)+u_{2}(Y) \dot{h}(X, Z),
\end{gathered}
$$

which may be called the Codazzi-Mainardi equation with respect to the SSRM connection. Here the connection $\hat{\nabla}$ is the van der Waerden-Bortolotti connection with respect to SSRM connection defined by

$$
\left(\hat{\nabla}_{X} \hat{h}\right)(Y, Z)=\nabla_{X}^{\perp}(\hat{h}(Y, Z))-\hat{h}\left(\dot{\nabla}_{X} Y, Z\right)-\hat{h}\left(Y, \dot{\nabla}_{X} Z\right) .
$$

For unit normal vector fields $\eta, \mu$ of $M$ in $\widetilde{M}$, we have from (3.10), (3.16) and (3.19)

$$
\begin{gathered}
\tilde{\vec{\nabla}}_{X} \dot{\vec{\nabla}}_{Y} \eta=-\dot{\nabla}_{X}\left(\dot{A}_{\eta} Y\right)-\hat{h}\left(X, \dot{A}_{\eta} Y\right)-\dot{A}_{\nabla_{Y}^{\perp}} X+\nabla_{X}^{\perp} \nabla_{Y}^{\perp} \eta-u_{2}(X) \nabla_{Y}^{\perp} \eta \\
-g\left(\widetilde{\nabla}_{X} Y, U_{2}^{\top}\right) \eta-g\left(Y, \widetilde{\nabla}_{X} U_{2}^{\top}\right) \eta-u_{2}(Y) \widetilde{\nabla}_{X} \eta-u_{2}(Y) u_{1}(\eta) X \\
+u_{2}(X) u_{2}(Y) \eta+u_{2}(Y) u_{2}(\eta) X \\
\dot{\vec{\nabla}}_{Y} \dot{\widetilde{\nabla}}_{X} \eta=-\dot{\nabla}_{Y}\left(\dot{A}_{\eta} X\right)-\hat{h}\left(Y, \hat{A}_{\eta} X\right)-\dot{A}_{\nabla_{X}^{\perp} \eta} Y+\nabla_{Y}^{\perp} \nabla_{X}^{\perp} \eta-u_{2}(Y) \nabla_{X}^{\perp} \eta
\end{gathered}
$$




$$
\begin{gathered}
-g\left(\widetilde{\nabla}_{Y} X, U_{2}^{\top}\right) \eta-g\left(X, \widetilde{\nabla}_{Y} U_{2}^{\top}\right) \eta-u_{2}(X) \widetilde{\nabla}_{Y} \eta-u_{2}(X) u_{1}(\eta) Y \\
+u_{2}(Y) u_{2}(X) \eta+u_{2}(X) u_{2}(\eta) Y
\end{gathered}
$$

and

$$
\stackrel{\widetilde{\nabla}}{[X, Y]} \eta=-\dot{A}_{\eta}[X, Y]+\nabla_{[X, Y]}^{\perp} \eta-u_{2}([X, Y]) \eta
$$

From (3.24), (3.25) and (3.26), it follows that

$$
\begin{gathered}
\dot{\widetilde{R}}(X, Y, \eta, \mu)=R^{\perp}(X, Y, \eta, \mu)-g\left(\hat{h}\left(X, \dot{A}_{\eta} Y\right), \mu\right)+g\left(\hat{h}\left(Y, \dot{A}_{\eta} X\right), \mu\right) \\
\quad-u_{2}(X) g\left(\nabla_{Y}^{\perp} \eta, \mu\right)+u_{2}(Y) g\left(\nabla_{X}^{\perp} \eta, \mu\right)-g\left(Y, \widetilde{\nabla}_{X} U_{2}^{\top}\right) g(\eta, \mu) \\
\quad+g\left(X, \widetilde{\nabla}_{Y} U_{2}^{\top}\right) g(\eta, \mu)-u_{2}(Y) g\left(\widetilde{\nabla}_{X} \eta, \mu\right)+u_{2}(X) g\left(\widetilde{\nabla}_{Y} \eta, \mu\right),
\end{gathered}
$$

which yields from $(2.1),(2.2),(2.3),(3.12)$ and (3.18)

$$
\begin{gathered}
\tilde{\widetilde{R}}(X, Y, \eta, \mu)=R^{\perp}(X, Y, \eta, \mu)-g\left(h\left(X, A_{\eta} Y\right), \mu\right)+g\left(h\left(Y, A_{\eta} X\right), \mu\right) \\
+g\left(X, \nabla_{Y} U_{2}^{\top}\right) g(\eta, \mu)-g\left(Y, \nabla_{X} U_{2}^{\top}\right) g(\eta, \mu) \\
=R^{\perp}(X, Y, \eta, \mu)+g\left(\left(A_{\mu} A_{\eta}-A_{\eta} A_{\mu}\right) X, Y\right) \\
+g\left(X, \nabla_{Y} U_{2}^{\top}\right) g(\eta, \mu)-g\left(Y, \nabla_{X} U_{2}^{\top}\right) g(\eta, \mu) \\
=R^{\perp}(X, Y, \eta, \mu)+g\left(\left[A_{\mu}, A_{\eta}\right] X, Y\right) \\
+g\left(X, \nabla_{Y} U_{2}^{\top}\right) g(\eta, \mu)-g\left(Y, \nabla_{X} U_{2}^{\top}\right) g(\eta, \mu)
\end{gathered}
$$

which may be called the Ricci equation with respect to the SSRM connection.

Example. Let $T^{n}$ be a torus embedded in $R^{2 n}$ as follows:

$$
T^{n}=\left\{\left(\operatorname{cost}_{1}, \operatorname{sint}_{1}, \operatorname{cost}_{2}, \operatorname{sint}_{2}, \ldots, \operatorname{cost}_{n}, \sin _{n}\right) \mid t_{i} \in R\right\} .
$$

Then we have for each point $p=\left(\operatorname{cost}_{1}, \operatorname{sint}_{1}, \operatorname{cost}_{2}, \operatorname{sint}_{2}, \ldots, \operatorname{cost}_{n}, \operatorname{sint}_{n}\right) \in$ $T^{n}$

$$
T_{p}\left(T^{n}\right)=\operatorname{span}\left\{e_{1}, e_{2}, \ldots, e_{n}\right\}
$$

and

$$
T_{p}\left(T^{n}\right)^{\perp}=\operatorname{span}\left\{e_{n+1}, e_{n+2}, \ldots, e_{2 n}\right\},
$$

where

$$
e_{i}=\left(0, \ldots, 0,-\operatorname{sint}_{i}, \operatorname{cost}_{i}, 0, \ldots, 0\right)
$$

and

$$
e_{i+n}=\left(0, \ldots, 0, \text { cost }_{i}, \operatorname{sint}_{i}, 0, \ldots, 0\right)
$$


for $i=1, \ldots, n$.

Taking a differentiation of the above ones with respect to the Riemannian connection $\widetilde{\nabla}$, we have for $i=1, \ldots, n$

$$
\widetilde{\nabla}_{e_{i}} e_{i}=-e_{i+n}
$$

and

$$
\widetilde{\nabla}_{e_{i}} e_{i+n}=e_{i}
$$

and the other cases are zero.

From the formula of Gauss and the above relations, it follows that

$$
\widetilde{\nabla}_{e_{i}} e_{i}=-e_{i+n}=\nabla_{e_{i}} e_{i}+h\left(e_{i}, e_{i}\right),
$$

which yields

$$
\nabla_{e_{i}} e_{i}=0
$$

and

$$
h\left(e_{i}, e_{i}\right)=-e_{i+n}
$$

for $i=1, \ldots, n$.

Similarly, we have

$$
\widetilde{\nabla}_{e_{i}} e_{j}=0=\nabla_{e_{i}} e_{j}+h\left(e_{i}, e_{j}\right)
$$

which implies

$$
\nabla_{e_{i}} e_{j}=0
$$

and

$$
h\left(e_{i}, e_{j}\right)=0
$$

for $i, j=1, \ldots, n$ and $i \neq j$.

Now we define the vector fields $U_{1}$ and $U_{2}$ in $R^{2 n}$ as follows:

For each point $p=\left(x_{1}, x_{2}, \ldots, x_{2 n-1}, x_{2 n}\right) \in R^{2 n}$,

$$
U_{1}=\left(x_{1}, x_{2}, \ldots, x_{2 n-1}, x_{2 n}\right)
$$

and

$$
U_{2}=\left(-x_{2}, x_{1}, \ldots,-x_{2 n}, x_{2 n-1}\right) .
$$

It is easy to see that on $T^{n}$, the tangential and normal components of $U_{1}$ and $U_{2}$ are

$$
U_{1}^{\top}=0, U_{1}^{\perp}=U_{1}
$$


and

$$
U_{2}^{\top}=U_{2}, U_{2}^{\perp}=0
$$

, respectively.

Therefore, we have from (3.11), (3.27), (3.28) and (3.29)

$$
\dot{\nabla}_{e_{i}} e_{i}=u_{1}\left(e_{i}\right) e_{i}-2 u_{2}\left(e_{i}\right) e_{i}+U_{2}
$$

and

$$
\dot{\nabla}_{e_{i}} e_{j}=u_{1}\left(e_{j}\right) e_{i}-u_{2}\left(e_{i}\right) e_{j}-u_{2}\left(e_{j}\right) e_{i}
$$

for $i, j=1, \ldots, n$ and $i \neq j$.

Furthermore, taking account of (3.13), (3.14) and the last identities, we get for $i, j=1, \ldots, n$ and $i \neq j$

$$
\dot{T}\left(e_{i}, e_{j}\right)=u_{1}\left(e_{j}\right) e_{i}-u_{1}\left(e_{i}\right) e_{j}
$$

and

$$
\left(\dot{\nabla}_{e_{i}} g\right)\left(e_{i}, e_{i}\right)=2 u_{2}\left(e_{i}\right)
$$

and

$$
\left(\dot{\nabla}_{e_{j}} g\right)\left(e_{i}, e_{i}\right)=2 u_{2}\left(e_{j}\right)
$$

and the other cases are zero.

Also, from (3.12), (3.27), (3.28) and (3.29) we have for $i=1, \ldots, n$

$$
\grave{h}\left(e_{i}, e_{i}\right)=-e_{i+n}-U_{1}
$$

and the other cases are zero.

Therefore, from (3.15), (3.27), (3.29) and the last identities, it follows that

$$
\dot{H}=-\frac{1}{n} \sum_{i=1}^{n} e_{i+n}-U_{1}
$$

\section{Acknowledgments}

This study was supported by 2014 Research Grant from Kangwon National University (No. 120142434). 


\section{References}

[1] N.S. Agashe, M.R. Chafle, A semi-symmetric non-metric connection in a Riemannian manifold, Indian J. Pure Appl. Math. 23 (1992), 399-409.

[2] N.S. Agashe, M.R. Chafle, On submanifolds of a Riemannian manifold with semi-symmetric metric connection, Tensor (N.S.) 50, No. 3 (1992), 208-212.

[3] O.C. Andonie, D. Smaranda, Certaines connexious semi-symetriques, Tensor (N.S.) 31 (1977), 8-12.

[4] B.Y. Chen, Geometry of submanifolds, Pure and Appl. Math. No. 22, Marcel Dekker, Inc., New York (1973).

[5] Y. Dogru, On some properties of submanifolds of a Riemannian manifold endowed with a semi-symmetric non-metric connection, An. St. Univ. Ovidius Constanta 19, No. 3 (2011), 85-100.

[6] T. Imai, Hypersurfaces of a Riemannian manifold with semi-symmetric metric connection, Tensor (N.S.) 23 (1972), 300-306.

[7] S. Kobayashi, K. Nomizu, Foundations of differential geometry, Interscience Tracts in Pure and Appl. Math. No. 15, Vol. II, Interscience, New York (1969).

[8] Y. Liang, On semi-symmetric recurrent-metric connection, Tensor (N.S.) 55 (1994), 107-112.

[9] Z. Nakao, Submanifolds of a Riemannian manifold with semi-symmetric metric connections, Proc. Amer. Math. Soc. 54 (1976), 261-266.

[10] C. Ozgur, On submanifolds of a Riemannian manifold with a semisymmetric non-metric connection, Kuwait J. Sci. Eng. 37 (2010), 17-30.

[11] J. Sengupta, U.C. De, On a type of semi-symmetric non-metric connection, Bull. Calcutta Math. Soc. 92, No. 5 (2000), 375-384.

[12] J. Sengupta, U.C. De, T.Q. Binh, On a type of semi-symmetric non-metric connection on a Riemannian manifold, Indian J. Pure Appl. Math. 31, No. 12 (2000), 1659-1670.

[13] M.M. Tripathi, A new connection in a Riemannian manifold, Int. Electron. J. Geom. 1 (2008), 15-24. 
[14] K. Yano, On semi-symmetric metric connection, Rev. Roum. Math. Pures et Appl. 15 (1970), 1579-1586. 